\title{
Disorder-induced orbital ordering in doped manganites
}

\author{
Sanjeev Kumar ${ }^{1,2}$ and Arno P. Kampf ${ }^{3}$ \\ ${ }^{1}$ Faculty of Science and Technology, University of Twente, P.O. Box 217, 7500 AE Enschede, The Netherlands \\ ${ }^{2}$ Instituut-Lorentz, for Theoretical Physics, Leiden University, P.O. Box 9506, 2300 RA Leiden, The Netherlands \\ ${ }^{3}$ Theoretical Physics III, Center for Electronic Correlations and Magnetism, Institute of Physics, University of Augsburg, \\ D-86135 Augsburg, Germany
}

(Received 29 January 2008; revised manuscript received 27 March 2008; published 25 April 2008)

\begin{abstract}
We study the effect of quenched disorder on the ordering of orbital and magnetic degrees of freedom in a two-dimensional, two-band double-exchange model for $e_{g}$ electrons coupled to Jahn-Teller distortions. By using a real-space Monte Carlo method, we find that disorder can induce a short-range ordering of the orbital degrees of freedom near $30 \%$ hole doping. The most striking consequence of this short-range ordering is a strong increase in the low-temperature resistivity. The real-space approach allows us to analyze the spatial patterns of the charge, orbital, and magnetic degrees of freedom and the correlations among them. The magnetism is inhomogeneous on the nanoscale in the short-range orbitally ordered state.
\end{abstract}

DOI: 10.1103/PhysRevB.77.134442

PACS number(s): 71.10.-w, 75.47.Lx, 81.16.Rf

\section{INTRODUCTION}

Hole-doped perovskite manganites $\mathrm{RE}_{1-x} \mathrm{AE}_{x} \mathrm{MnO}_{3}$ ( $\mathrm{RE}$ $=$ rare earth, $\mathrm{AE}=$ alkaline earth) have attracted great attention from the condensed matter community over the past decade. ${ }^{1}$ While the initial surge of research activities on these materials was triggered by the discovery of the colossal magnetoresistance effect, a rich variety of phases and phase transitions was subsequently uncovered. ${ }^{2,3}$ It is now widely accepted that the interplay among charge, spin, orbital, and lattice degrees of freedom is the underlying cause of the complexity and richness of the physical phenomena observed in manganites. Recent efforts from both experiment and theory have highlighted the significance of quenched disorder in these materials. ${ }^{4-7}$ Therefore, analyzing the effects of disorder in manganites has become an active area of research. ${ }^{8-11}$

Disorder is generally viewed as an agent for suppressing the ordering tendencies of the microscopic degrees of freedom. Experiments on the half-doped $(x=0.5)$ manganites show that quenched disorder indeed spoils the long-range ordering of the charge, orbital, and spin variables leading, in some cases, to a short-range ordering of these microscopic degrees of freedom. ${ }^{5,6}$ The opposite effect, however, is observed in manganites near 30\% hole doping, where an ordering of the orbital degrees of freedom is induced by the presence of quenched disorder. ${ }^{7}$

In manganites, the average $r_{A}$ and the variance $\sigma^{2}$ of the $A$-site ionic radii are known to control the single-particle bandwidth and the magnitude of quenched disorder, respectively. ${ }^{12}$ Samples with constant $r_{A}$ and varying $\sigma^{2}$ were used in the experiments of Ref. 7 with a combination of $\mathrm{La}$, $\mathrm{Pr}, \mathrm{Nd}$, and $\mathrm{Sm}$ and $\mathrm{Ca}, \mathrm{Sr}$, and $\mathrm{Ba}$ at the $A$ site, while keeping $x=0.3$. An increase in the low-temperature resistivity by 4 orders of magnitude was attributed to the onset of orbital ordering, which was also evidenced from the structural changes analyzed via powder x-ray diffraction. Magnetism is strongly affected with a reduction in both the Curie temperature $T_{C}$ and the saturation value of the magnetization. This doping regime is also believed to be magnetically inho- mogeneous, as independently inferred from NMR and neutron scattering experiments. ${ }^{13,14}$

Disorder has been previously included in models for manganites to study its influence on the long-range ordered phases $8,15,16$ especially near a first-order phase boundary or in the vicinity of phase separation. ${ }^{9,11,17}$ The idea that quenched disorder may lead to a partial ordering of the orbital degrees of freedom in manganite models has so far remained unexplored.

In this paper, we study a two-band double-exchange model with quenched disorder by using a real-space Monte Carlo method. Disorder is modeled via random on-site energies selected from a given distribution. We consider two different types of distributions, which are described in detail in the next section. Here and below, we refer to these distributions as (i) binary disorder and (ii) random scatterers. While the binary disorder has no significant effect on the orbital degrees of freedom, random scatterers lead to orbitally ordered regions, and a sharp increase in the low-temperature resistivity is found, as observed in the experiment. ${ }^{7}$ The magnetic structure is inhomogeneous in a restricted doping regime, as observed in the NMR and the neutron scattering experiments. Within clusters, staggered orbital ordering is accompanied by ferromagnetism, thus providing an example of Goodenough-Kanamori rules in an inhomogeneous system. ${ }^{18,19}$

\section{MODEL AND METHOD}

We consider a two-band model for itinerant $e_{g}$ electrons on a square lattice. The electrons are coupled to Jahn-Teller (JT) lattice distortions, $t_{2 g}$-derived $S=3 / 2$ core spins, and quenched disorder, as described by the Hamiltonian,

$$
\begin{aligned}
H= & \sum_{\langle i j\rangle \sigma}^{\alpha \beta} t_{\alpha \beta}^{i j} c_{i \alpha \sigma}^{\dagger} c_{j \beta \sigma}+\sum_{i} \epsilon_{i} n_{i}+J_{s} \sum_{\langle i j\rangle} \mathbf{S}_{i} \cdot \mathbf{S}_{j}-J_{H} \sum_{i} \mathbf{S}_{i} \cdot \boldsymbol{\sigma}_{i} \\
& -\lambda \sum_{i} \mathbf{Q}_{i} \cdot \boldsymbol{\tau}_{i}+\frac{K}{2} \sum_{i} \mathbf{Q}_{i}^{2} .
\end{aligned}
$$

Here, $c$ and $c^{\dagger}$ are annihilation and creation operators for $e_{g}$ 
electrons, $\sigma=\uparrow, \downarrow$ is the spin index, and $\alpha, \beta$ are summed over the two Mn $e_{g}$ orbitals $d_{x^{2}-y^{2}}$ and $d_{3 z^{2}-r^{2}}$, which are labeled $(a)$ and $(b)$ in what follows. $t_{\alpha \beta}^{i j}$ denote the hopping amplitudes between $e_{g}$ orbitals on nearest-neighbor sites and have the cubic perovskite specific forms $t_{a a}^{x}=t_{a a}^{y} \equiv t, t_{b b}^{x}=t_{b b}^{y}$ $\equiv t / 3, t_{a b}^{x}=t_{b a}^{x} \equiv-t / \sqrt{3}$, and $t_{a b}^{y}=t_{b a}^{y} \equiv t / \sqrt{3}$, where $x$ and $y$ mark the spatial directions. ${ }^{20}$ The $e_{g}$-electron spin is locally coupled to the $t_{2 g}$ spin $\mathbf{S}_{i}$ via the Hund's rule coupling $J_{H}$. The $e_{g}$-electron spin is given by $\sigma_{i}^{\mu}=\sum_{\sigma \sigma^{\prime}}^{\alpha} c_{i \alpha \sigma}^{\dagger} \Gamma_{\sigma \sigma^{\prime}}^{\mu} c_{i \alpha \sigma^{\prime}}$, where $\Gamma^{\mu}$ are the Pauli matrices. $J_{s}$ is the strength of the superexchange coupling between neighboring $t_{2 g}$ spins. $\lambda$ denotes the strength of the JT coupling between the distortion $\mathbf{Q}_{i}=\left(Q_{i x}, Q_{i z}\right)$ and the orbital pseudospin $\tau_{i}^{\mu}$ $=\Sigma_{\sigma}^{\alpha \beta} c_{i \alpha \sigma}^{\dagger} \Gamma_{\alpha \beta}^{\mu} c_{i \beta \sigma} . K$ is a measure of the lattice stiffness, and we set $t=1=K$ as our reference energy scale.

The following two forms of on-site disorder modeling are used: (i) binary disorder, $\epsilon_{i}$ takes equally probable values $\pm \Delta$; (ii) random scatterers, a fraction $x$ of the sites are taken to have $\epsilon_{i}=D$, while for the other sites, $\epsilon_{i}=0$. Although the first choice of disorder is the simplest from the model point of view, the second appears more realistic. In real materials, a fraction $x$ of the rare-earth ions is replaced by alkalineearth ions at random locations. Therefore, it is likely that the disorder arising as a consequence of this substitution is connected to the amount of doping. This situation is modeled by placing repulsive potentials on a fraction $x$ of the sites, which are randomly selected. A typical measure of the strength of a disorder distribution is its variance. For the binary distribution, the variance is $\Delta$, while for the finite density $x$ of scatterers with potential strength $D$, it is $D \sqrt{x(1-x)}$. These two models for disorder were previously employed in a study of half-doped manganites. ${ }^{11}$ The JT distortions and the $t_{2 g}$ derived core spins are treated as classical variables, and we set $\left|\mathbf{S}_{i}\right|=1$. Guided by earlier estimates for the JT coupling strength in manganites, we fix $\lambda=1.5$ (Ref. 21) and explore the variation in the parameters $\Delta, D$, and $J_{s}$.

We further adopt the simplifying limit $J_{H} \gg t$, which is justified and frequently used in the context of manganites..$^{9,20,22}$ In this limit, the electronic spin at site $i$ is tied to the orientation of the core spin $\mathbf{S}_{i}$. Transforming the fermionic operators to this local spin reference frame leads to the following effectively "spinless" model for the $e_{g}$ electrons:

$$
\begin{aligned}
H= & \sum_{\langle i j\rangle}^{\alpha \beta} \tilde{t}_{\alpha \beta}^{i j} c_{i \alpha}^{\dagger} c_{j \beta}+\sum_{i} \epsilon_{i} n_{i}+J_{s} \sum_{\langle i j\rangle} \mathbf{S}_{i} \cdot \mathbf{S}_{j}-\lambda \sum_{i} \mathbf{Q}_{i} \cdot \boldsymbol{\tau}_{i} \\
& +\frac{K}{2} \sum_{i} \mathbf{Q}_{i}^{2} .
\end{aligned}
$$

The new hopping amplitudes $\tilde{t}$ have an additional dependence on the core-spin configurations and are given by

$$
\frac{\tilde{t}_{\alpha \beta}}{t_{\alpha \beta}}=\cos \frac{\theta_{i}}{2} \cos \frac{\theta_{j}}{2}+\sin \frac{\theta_{i}}{2} \sin \frac{\theta_{j}}{2} e^{-i\left(\phi_{i}-\phi_{j}\right)} .
$$

Here, $\theta_{i}$ and $\phi_{i}$ denote polar and azimuthal angles for the spin $\mathbf{S}_{i}$. From now on, the operator $c_{i \alpha}\left(c_{i \alpha}^{\dagger}\right)$ is associated

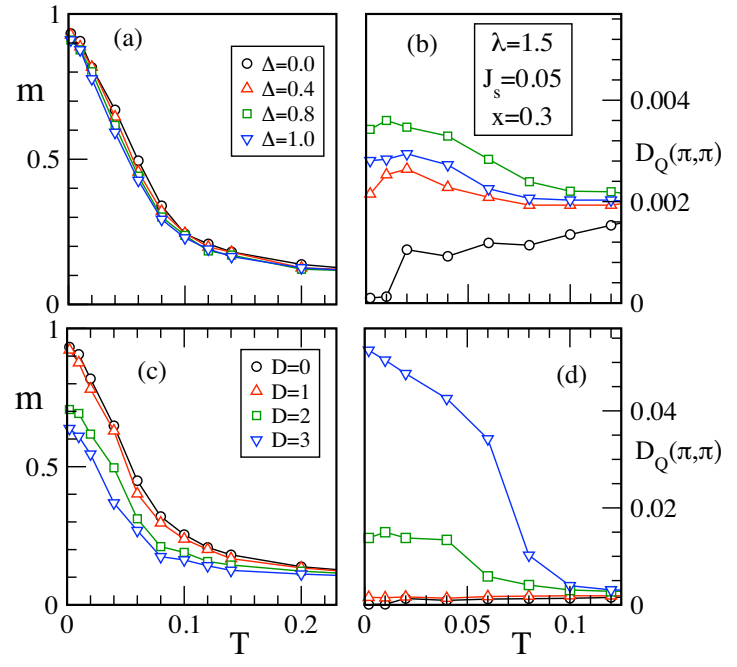

FIG. 1. (Color online) Temperature dependence of (a) the magnetization $m$ and (b) the lattice structure factor $D_{Q}(\mathbf{q})$ at $\mathbf{q}=(\pi, \pi)$ for various values of the disorder strength $\Delta$. (c) and (d) show the same quantities as in (a) and (b), respectively, if the on-site disorder is modeled by random scatterers of strength $D$. The concentration of scatterers is equal to the hole density, $x=0.3$. Note the order of magnitude difference in the magnitudes for $D_{Q}\left(\mathbf{q}_{0}\right)$ between panels (b) and (d). All results are at $\lambda=1.5$ and $J_{s}=0.05$.

with annihilating (creating) an electron at site $i$ in the orbital $\alpha$ with spin parallel to $\mathbf{S}_{i}$.

The model given by Eq. (2) is bilinear in the electronic operators and does not encounter the problem of an exponentially growing Hilbert space since all many-particle states can be constructed from Slater determinants of the singleparticle states. The difficulty, however, arises from the large phase space in the classical variables $\mathbf{Q}$ and $\mathbf{S}$. Exact diagonalization (ED) based Monte Carlo is a numerically exact method to treat such problems, and has been extensively used in the past. ${ }^{9,20,22}$ The classical variables are sampled by the Metropolis algorithm, which requires the exact eigenenergy spectrum. Therefore, iterative ED of the Hamiltonian is needed, which leads to $N^{4}$ scaling of the required CPU time, where $N$ is the number of lattice sites. The $N^{4}$ scaling makes this method very restrictive in terms of the achievable lattice sizes, with the typical size in previous studies being $\sim 100$ sites. Since a study of larger lattices is essential for analyzing the nature of inhomogeneities in manganite models, several attempts have been made to devise accurate approximate schemes. ${ }^{23-25}$ In the present study, we employ the traveling cluster approximation, ${ }^{25}$ which indeed has been very successful in analyzing similar models in the recent past. ${ }^{10,11,26}$

\section{RESULTS AND DISCUSSION}

We begin with the results for bulk quantities describing the ordering of the magnetic and the lattice degrees of freedom. We focus on the $30 \%$ hole-doped system $(x=0.3)$ for a close correspondence to the experiments in Ref. 7. Figure 1(a) shows the effect of binary disorder on the temperature dependence of the magnetization $m$ defined via $m^{2}$ $=\left\langle\left(N^{-1} \Sigma \mathbf{S}_{i}\right)^{2}\right\rangle_{a v}$. Here and below, $\langle\cdots\rangle_{\text {av }}$ denotes the average 
over thermal equilibrium configurations and additionally over realizations of quenched disorder. Results for disordered systems are averaged over four to six realizations of disorder. Clearly, the magnetism is not affected much by the presence of weak binary disorder. This is in agreement with previous studies, which find that the reduction in $T_{C}$ is proportional to $\Delta^{2}$ for weak disorder. ${ }^{27-29}$

Figure 1(b) shows the temperature dependence of the $\mathbf{q}$ $=\mathbf{q}_{0} \equiv(\pi, \pi)$ component of the lattice structure factor, $D_{Q}(\mathbf{q})=N^{-2} \sum_{i j}\left\langle\mathbf{Q}_{i} \cdot \mathbf{Q}_{j}\right\rangle_{a v} e^{-i \mathbf{q} \cdot\left(\mathbf{r}_{i}-\mathbf{r}_{j}\right)} . D_{Q}\left(\mathbf{q}_{0}\right)$ is a measure for the staggered distortion order in the system. The lattice ordering leads to orbital ordering via the JT coupling. An increase with $\Delta$ in the low-temperature value of $D_{Q}\left(\mathbf{q}_{0}\right)$ suggests the appearance of orbital order. However, this effect is too weak to explain the experimental resistivity data. ${ }^{7}$ Moreover, the increase at low $T$ in $D_{Q}\left(\mathbf{q}_{0}\right)$ is not monotonic, which becomes clear by comparing the results for $\Delta=0.4$, 0.8 , and 1.0 in Fig. 1(b).

Now, we explore the results for the disorder arising from random scatterers of strength $D$. Since the disorder originates from the replacement of $\mathrm{RE}^{3+}$ by $\mathrm{AE}^{2+}$ ions, the density of random scatterers is kept equal to the doping concentration $x$. Since the two models for binary disorder and random scatterers, respectively, are identical at $x=0.5$, the two models are compared for $x<0.5 . m(T)$, shown in Fig. 1(c), is affected strongly upon increasing $D$, with a decrease in the saturation value of the magnetization pointing toward a magnetically inhomogeneous ground state. More importantly, a monotonic increase with $D$ is observed in the lowtemperature values of $D_{Q}\left(\mathbf{q}_{0}\right)$ [see Fig. 1(d)]. The rise in $D_{Q}\left(\mathbf{q}_{0}\right)$ clearly indicates the emergence of orbital ordering in the system, with the area and/or strength of the ordered regions increasing with increasing $D$.

It is expected that these orbital ordering correlations are reflected in the transport properties. We therefore compute the dc resistivity $\rho$ approximated by the inverse of lowfrequency optical conductivity, which is calculated by using exact eigenstates and eigenenergies in the Kubo-Greenwood formula. ${ }^{30}$ Figures 2(a) and 2(b) show $\rho$ as a function of temperature for the two disorder models described above. The low-temperature resistivity increases upon increasing the binary disorder strength $\Delta$ [see Fig. 1(a)]. For small values of $\Delta$, the resistivity curves appear parallel to each other below $T \sim 0.1$. The resistivity therefore follows Mathiessen's rule, i.e., $\rho(T)$ for the disordered system is obtained from $\rho(T)$ for the clean system by simply adding a constant contribution arising from the scattering off the disorder potential. $d \rho / d T$ remains positive at low temperature, indicating a metallic behavior. This oversimplified description, however, does not take into account the disorder-induced changes in the orbital ordering correlations and the related changes in the density of states (discussed below).

Random scatterers lead to a drastically different behavior. The low-temperature rise in $\rho(T)$ covers several orders of magnitude [see Fig. 2(b)]. The negative sign of $d \rho / d T$ for $D>1$ signals an insulating behavior. Upon increasing the disorder strength $D$, we therefore observe a metal to insulator transition. For $x=0.3$, both disorder models have the same variance if $\Delta \sim 0.46 D$ holds. Comparing, therefore, the results for $D=2$ and $\Delta=1$, we have to conclude that the drastic
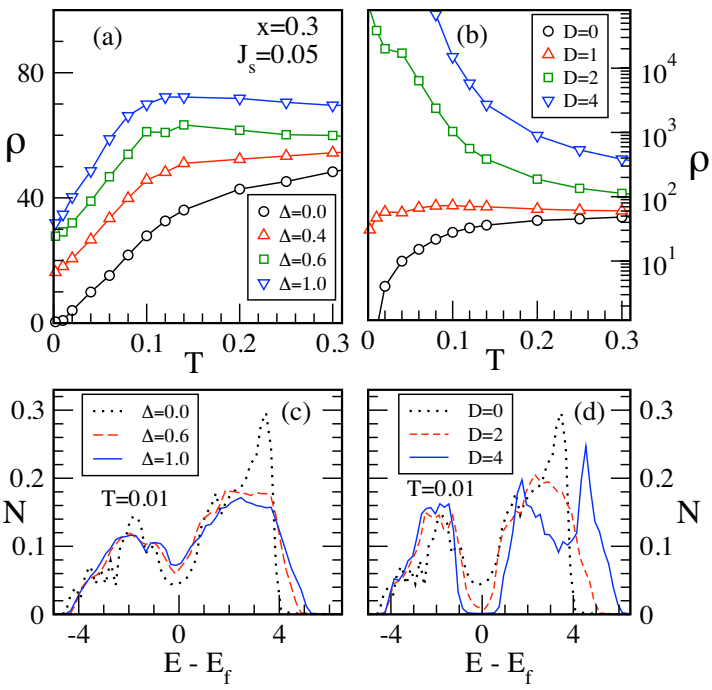

FIG. 2. (Color online) Temperature dependent resistivity $\rho(T)$ (in units of $\hbar / \pi e^{2}$ ) for varying strength of (a) binary disorder $\Delta$ and (b) random scatterers $D$. Note the logarithmic scale in (b). [(c) and (d) Low-temperature density of states for the two types of disorder.

rise in the resistivity for random scatterers cannot be attributed to the strength of the disorder potential. In fact, a large increase in the low- $T$ resistivity was one of the experimental indications for the onset of disorder-induced orbital ordering. ${ }^{7}$

Figures 2(c) and 2(d) highlight the difference between the densities of states (DOSs) for the two choices of disorder modeling. The DOS is defined as $N(\omega)=\left\langle N^{-1} \sum_{i} \delta\left(\omega-E_{i}\right)\right\rangle_{a v}$, where $E_{i}$ denotes the eigenvalues of the Hamiltonian. Here, we approximate the $\delta$ function by a Lorentzian with width $\gamma=0.04$,

$$
\delta\left(\omega-E_{i}\right) \simeq \frac{\gamma / \pi}{\left[\gamma^{2}+\left(\omega-E_{i}\right)^{2}\right]} .
$$

The DOS for the clean system has a pseudogap structure near the chemical potential. For binary disorder, the pseudogap slowly fills up with increasing $\Delta$. In contrast, it deepens upon adding random scattering centers and even leads to a clean
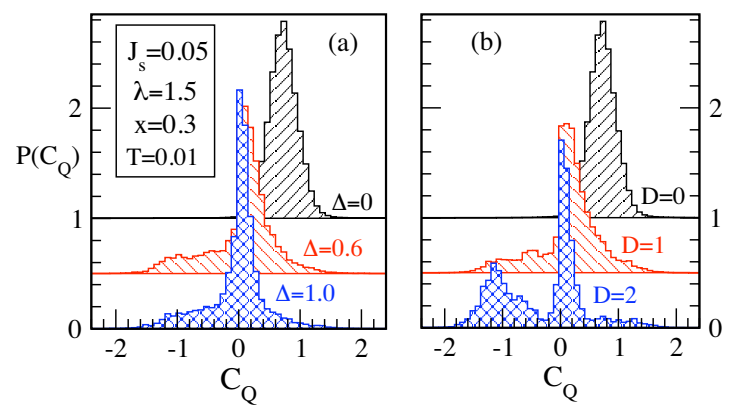

FIG. 3. (Color online) Low-temperature distribution functions generated from the Monte Carlo data for the nearest-neighbor correlations $C_{Q}$ of the lattice distortions for (a) binary disorder and (b) for random scatterers. The curves for different $\Delta$ are off-set along the $y$ axis for clarity. $C_{Q}$ is positive (negative) for ferro(antiferro)distortive patterns of the lattice variables. 
$\varepsilon(\mathrm{i})$

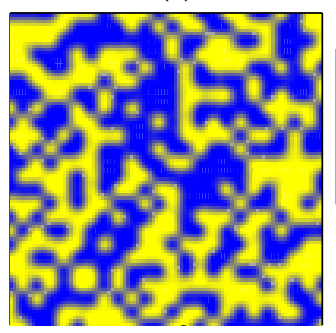

$\varepsilon(i)$

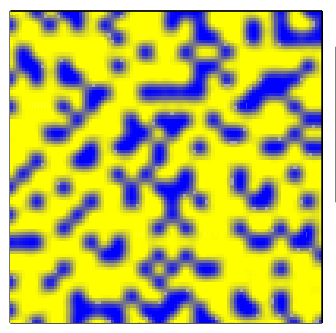

$\mathrm{n}(\mathrm{i})$

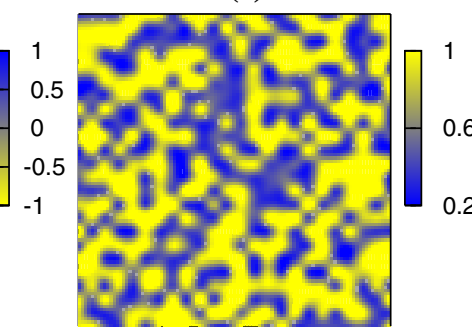

$\mathrm{n}(\mathrm{i})$

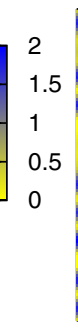

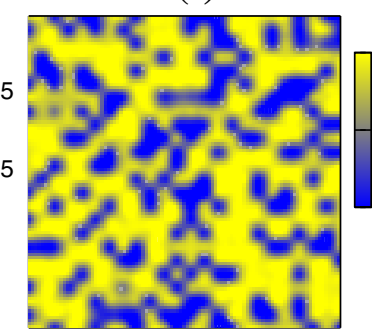

$\mathrm{C}_{\mathrm{Q}}(\mathrm{i})$

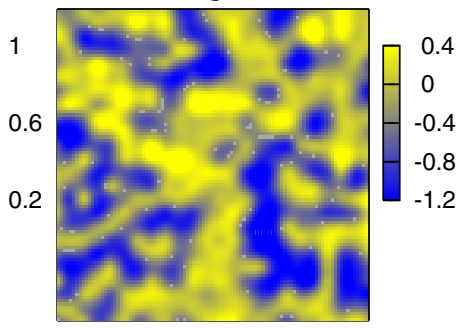

FIG. 4. (Color online) Realspace patterns of the disorder potential $\epsilon(i)$, charge density $n(i)$, and lattice correlations $C_{Q}(i)$. Top row: binary disorder with $\Delta=1$; bottom row: random scatterers with $D=2$. The patterns in both cases are shown on a $24 \times 24$ lattice for a single disorder realization at $T=0.01$ and $x=0.3$. gap for $D \geqslant 3$. This opposite behavior is partly responsible for the drastically different low-temperature resistivity discussed above. The three-peak structure for large values of $D$ in Fig. 2(d) can be understood as follows: a fraction $2 x$ of electronic states split off and form a narrow impurity band centered at an energy $D$ above the Fermi level of the undoped system. The lower band now contains a fraction 2(1 $-x)$ of the states with the Fermi level located in the middle of the band. This leads to a situation similar to the undoped system, and an energy gap originating from staggered orbital ordering opens at the Fermi level.

To gain further insight into the nature of the states in the presence of the two types of disorder, we plot the distribution functions for the lattice variables in Fig. 3. Panel (a) shows the distribution of the nearest-neighbor lattice correlations, $C_{Q}(i)=(1 / 4) \sum_{\delta} \mathbf{Q}_{i} \cdot \mathbf{Q}_{i+\delta}$ for binary disorder; here, $\delta$ denotes the four nearest-neighbor sites of site $i$. A negative value of $C_{Q}(i)$ indicates an antiferro pattern of JT distortions and, hence, a pattern of staggered orbital ordering. The distribution function for $C_{Q}$ is defined as $P\left(C_{Q}\right)=\left\langle N^{-1} \sum_{i} \delta\left[C_{Q}\right.\right.$ $\left.\left.-C_{Q}(i)\right]\right\rangle_{a v}$; the $\delta$ function is again approximated by a Lorentzian with width $\sim 0.04$. A peak in $P\left(C_{Q}\right)$ centered near $C_{Q}=0.8$ for $\Delta=0$ indicates that the clean system has weak ferrodistortive and hence ferro-orbital correlations. Tails going down to $C_{Q} \sim-1.4$ arise in the distribution function upon including binary disorder.

The distribution function $P\left(C_{Q}\right)$ for random scatterers looks qualitatively different. We recall that the strengths of the two types of disorder are related via $\Delta \sim 0.46 D$. The lowtemperature distributions $P\left(C_{Q}\right)$ are plotted in Fig. 3(b) for random scatterers. A qualitative change in the shape of the distribution function occurs for $D=2$, where a second peak
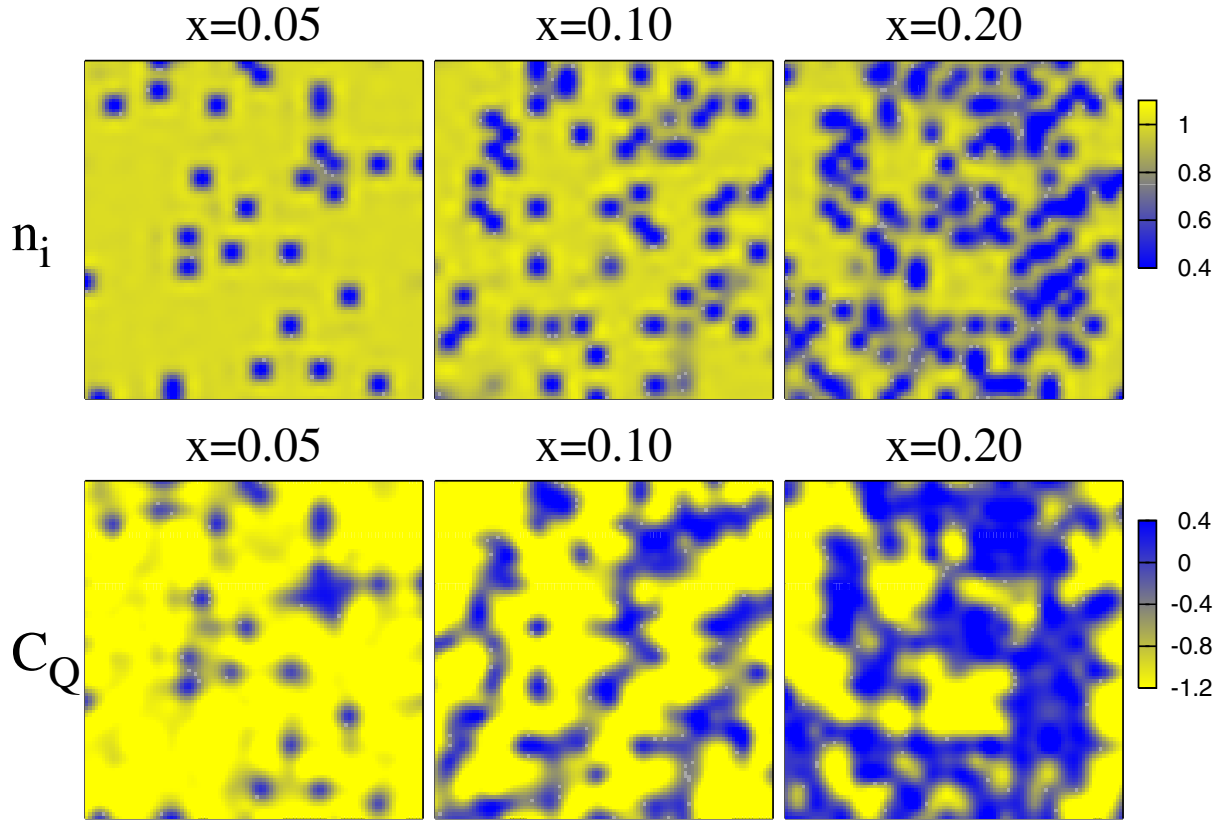

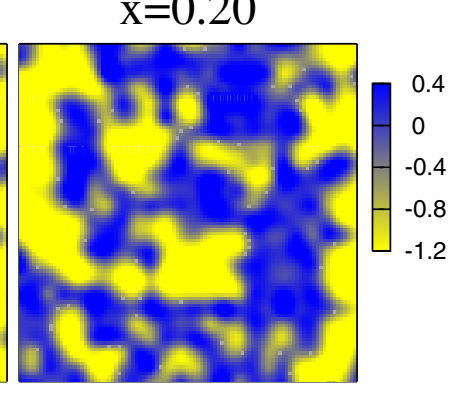

FIG. 5. (Color online) Doping evolution of the local charge density $n_{i}$ and the local lattice correlations $C_{Q}(i)$ for a single realization of random scatterers with strength $D=1$ on a $24 \times 24$ lattice. 

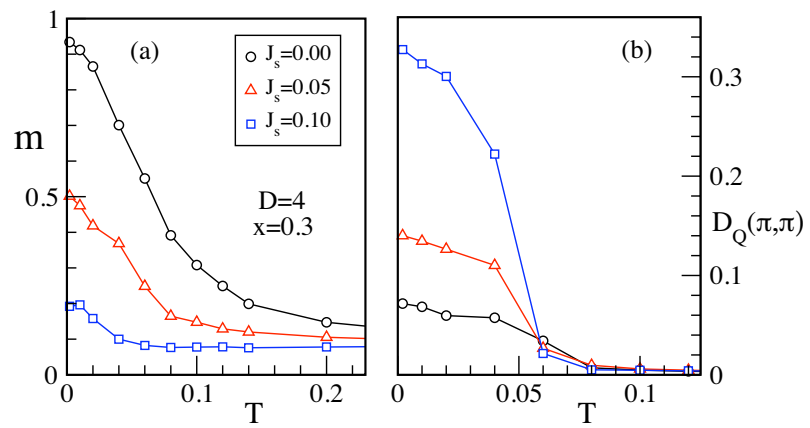

FIG. 6. (Color online) Temperature dependence of the magnetization $m$ and the staggered lattice structure factor $D_{Q}(\pi, \pi)$ for varying superexchange coupling strength $J_{s}$. The results are for random scatterers with strength $D=4$.

centered around $C_{Q} \sim-1.2$ emerges. This is a direct indication that a significant fraction of the system becomes orbitally ordered. This perfectly correlates with the strong rise in $D_{Q}\left(\mathbf{q}_{0}\right)$ at low temperatures [see Fig. 1(b)] and the anomalous increase in the resistivity [see Fig. 2(b)].

A real-space picture for the emergence of orbital ordering is presented in Fig. 4, which displays the disorder potential $\epsilon_{i}$, the electronic density $n_{i}$, and the lattice correlations $C_{Q}(i)$. The top row for binary disorder shows that the charge density closely follows the disorder potential. The local lattice correlations are centered around $C_{Q}=0$, which is also evident from the peak in the distribution $P\left(C_{Q}\right)$ shown in Fig. 3(a). The bottom row in Fig. 4 shows the corresponding results for the disorder potential arising from random scatterers. Since the doping concentration in this case coincides with the concentration of the scatterers, the holes are trapped at the impurity sites. This leaves the surrounding effectively undoped and thereby induces orbital ordering. This is apparent from the spread of the dark-blue regions and their cross correlation with the charge density distribution in the bottom row of Fig. 4. Such a picture with orbitally ordered regions coexisting with orbitally disordered patches perfectly describes the double peak structure of the distribution function in Fig. 3(b).

Although we are primarily interested in the experimentally relevant case $x=0.3$, it is useful to see how the realspace patterns evolve as one moves from low to high hole densities. The undoped system is an orbitally ordered insulator, which turns into an orbitally disordered metal upon doping. ${ }^{31}$ We show real-space patterns at three different doping concentrations in Fig. 5. The density of random scatterers is kept equal to the doping fraction $x$. The charge density distribution is largely controlled by the disorder distribution. At low doping, disconnected orbitally disordered regions are essentially tied to the trapped holes. With increasing $x$, the orbitally disordered regions begin to connect in onedimensional snakelike patterns. By further increasing the doping and the concentration of scattering centers, the orbitally disordered regions grow. Since the low-doping regime of the present model is phase separated, ${ }^{1,10}$ the phenomenon of disorder-induced orbital ordering occurs only for $x>0.25$. The upper critical value of doping concentration, beyond which this phenomenon does not occur, depends on the strength of the disorder potential used. The inhomogeneous structures shown in Fig. 5 arise from the combined effects of disorder and phase separation tendencies.

It is worthwhile to point to a similarity between the effects of disorder in the present study and in a model analysis for $d$-wave superconductors with nonmagnetic impurities. In Ref. 32, it was found that the impurities nucleate antiferromagnetism in their near vicinity. Upon increasing the impurity concentration, static antiferromagnetism is observed. There seems to be a perfect analogy between the two situations if one interchanges antiferromagnetism by orbital ordering; both are ordering phenomena with the staggered ordering wave vector $(\pi, \pi)$. The $(\pi, \pi)$ ordering phenomena are partially triggered by the charge inhomogeneities in both cases. An additional complication in the present case arises from the spin degrees of freedom in addition to the orbital variables and from the anisotropy of the hopping parameters.

$$
\mathrm{J}_{\mathrm{s}}=0.02
$$
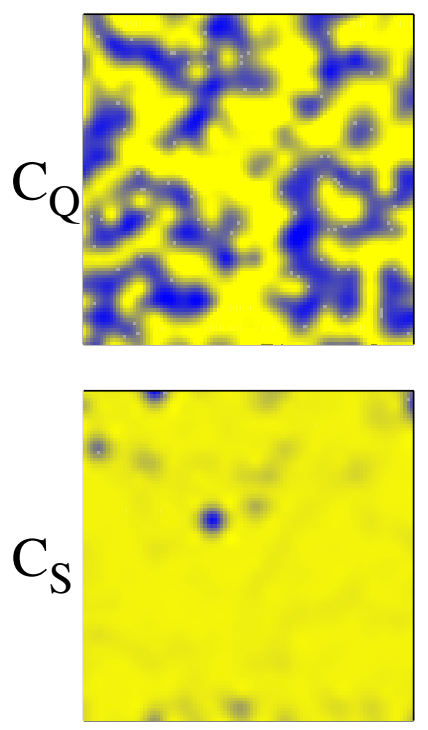

$\mathrm{J}_{\mathrm{s}}=0.06$
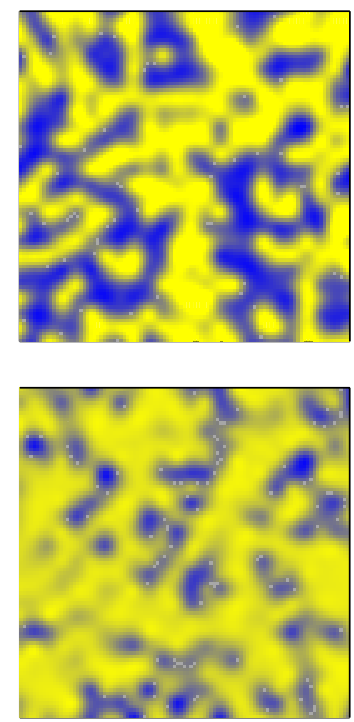

$\mathrm{J}_{\mathrm{S}}=0.10$
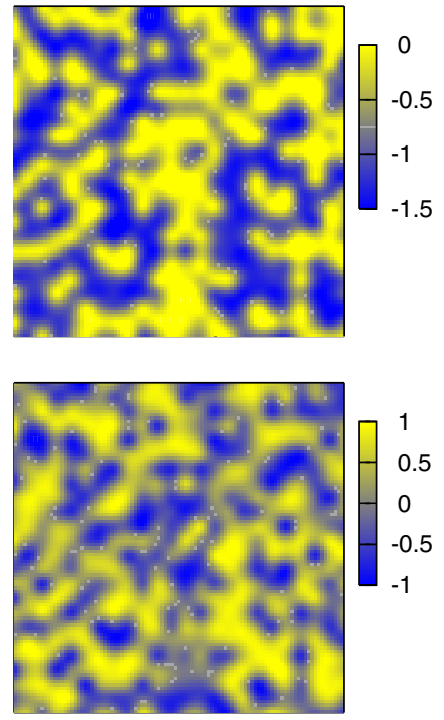

FIG. 7. (Color online) Realspace patterns for the lattice correlations $C_{Q}(i)$ and the analogously defined spin correlations $C_{S}(i)$ for varying superexchange coupling $J_{s}$. The patterns are shown for a single disorder realization at $T$ $=0.01$. Orbitally ordered regions tend to maintain ferromagnetism, while the orbitally disordered regions are more susceptible toward antiferromagnetism with increasing $J_{s}$. 
As inferred above from the results for the temperature dependent magnetization $m(T)$, the magnetic ground state appears to be homogeneous for binary disorder but may be inhomogeneous in the case of doped scatterers [see Figs. 1(a) and 1(c)]. Since the magnetism is partially controlled by the antiferromagnetic superexchange coupling $J_{s}$, we study the effect of increasing $J_{s}$ for a fixed large disorder strength of random scatterers. Figure 6(a) shows the result for $m(T)$ and Fig. 6(b) shows the result for the temperature dependence of $D_{Q}\left(\mathbf{q}_{0}\right)$. The saturation value of $m(T)$ as well as the onset scale for ferromagnetism decrease with increasing $J_{s}$. More importantly, $D_{Q}\left(\mathbf{q}_{0}\right)$ at low temperatures increases with increasing $J_{s}$, indicating an enhancement in the orbital ordering. For a homogeneous system, this would mean that orbital ordering and antiferromagnetism are both enhanced with increasing $J_{s}$. This is a contradiction to the GoodenoughKanamori rules, which state that an orbitally antiferro system should be magnetically ferro. The contradiction is resolved by analyzing the microscopic details of this complicated state providing an example where the real-space structures are essential for a comprehensive understanding.

We show in Fig. 7 the effect of the superexchange coupling on the real-space patterns of lattice and spin variables. The lattice correlations are shown in the top row, and the analogously defined spin correlations, $C_{S}(i)$ $=(1 / 4) \sum_{\delta} \mathbf{S}_{i} \cdot \mathbf{S}_{i+\delta}$, in the bottom row. For $J_{s}=0.02$, the system contains orbitally ordered nanoscale regions, but magnetically, it appears homogeneous. For $J_{s}=0.06$, the area of the orbitally ordered regions is enlarged and magnetic inhomo- geneities appear. The orbitally ordered regions remain ferromagnetic, while the orbitally disordered regions become antiferromagnetic upon increasing $J_{s}$. In the orbitally ordered clusters of this inhomogeneous system in the selected parameter regime, the Goodenough-Kanamori rules are therefore fulfilled. However, upon increasing $J_{s}$ further to 0.1 , the antiferromagnetic regions start to extend also into the orbitally ordered clusters. The charge density patterns (not shown here) are insensitive to the increase in $J_{s}$.

\section{CONCLUSIONS}

Our analysis for a two-band double-exchange model for manganites leads us to conclude that the disorder-induced orbital ordering in manganites near $x=0.3$ is properly described if the density of scattering centers tracks the hole concentration. Within this specific model of quenched disorder, the induced staggered orbital ordering is responsible for the orders of magnitude increase in the low-temperature resistivity, as observed in the experiments in Ref. 7.

\section{ACKNOWLEDGMENTS}

S.K. acknowledges support by "NanoNed," a nanotechnology programme of the Dutch Ministry of Economic Affairs. A.P.K. gratefully acknowledges support by the Deutsche Forschungsgemeinschaft through SFB 484. Simulations were performed on the Beowulf Cluster at HRI, Allahabad (India).
${ }^{1}$ For overviews, see Nanoscale Phase Separation and Colossal Magnetoresistance, edited by E. Dagotto (Springer-Verlag, Berlin, 2002) and Refs. 2 and 3.

${ }^{2}$ Colossal Magnetoresistive Oxides, edited by T. Chatterji (Kluwer, Dordrecht, 2004).

${ }^{3}$ Colossal Magnetoresistive Oxides, edited by Y. Tokura (Gordon and Breach, Amsterdam, 2000).

${ }^{4}$ L. M. Rodriguez-Martinez and J. P. Attfield, Phys. Rev. B 58, 2426 (1998); 54, R15622 (1996).

${ }^{5}$ D. Akahoshi, M. Uchida, Y. Tomioka, T. Arima, Y. Matsui, and Y. Tokura, Phys. Rev. Lett. 90, 177203 (2003).

${ }^{6}$ Y. Tomioka and Y. Tokura, Phys. Rev. B 70, 014432 (2004).

${ }^{7}$ L. M. Rodriguez-Martinez and J. P. Attfield, Phys. Rev. B 63, 024424 (2000).

${ }^{8}$ G. Alvarez, H. Aliaga, C. Sen, and E. Dagotto, Phys. Rev. B 73, 224426 (2006).

${ }^{9}$ A. Moreo, M. Mayr, A. Feiguin, S. Yunoki, and E. Dagotto, Phys. Rev. Lett. 84, 5568 (2000).

${ }^{10}$ S. Kumar, A. P. Kampf, and P. Majumdar, Phys. Rev. Lett. 97, 176403 (2006).

${ }^{11}$ K. Pradhan, A. Mukherjee, and P. Majumdar, Phys. Rev. Lett. 99, 147206 (2007).

${ }^{12}$ For precise definitions of $r_{A}$ and $\sigma^{2}$, see, e.g., J. P. Attfield, Int. J. Inorg. Mater. 3, 1147 (2001).

${ }^{13}$ G. Papavassiliou, M. Pissas, M. Belesi, M. Fardis, J. Dolinsek, C. Dimitropoulos, and J. P. Ansermet, Phys. Rev. Lett. 91,
147205 (2003)

${ }^{14}$ M. Hennion, F. Moussa, G. Biotteau, J. Rodriguez-Carvajal, L. Pinsard, and A. Revcolevschi, Phys. Rev. Lett. 81, 1957 (1998).

${ }^{15}$ Y. Motome, N. Furukawa, and N. Nagaosa, Phys. Rev. Lett. 91, 167204 (2003).

${ }^{16}$ C. Sen, G. Alvarez, and E. Dagotto, Phys. Rev. B 70, 064428 (2004).

${ }^{17}$ S. Kumar and P. Majumdar, Phys. Rev. Lett. 92, 126602 (2004).

${ }^{18} \mathrm{~J}$. B. Goodenough, Magnetism and the Chemical Bond (Interscience, New York, 1963); J. Kanamori, J. Phys. Chem. Solids 10, 87 (1959).

${ }^{19}$ M. Imada, A. Fujimori, and Y. Tokura, Rev. Mod. Phys. 70, 1039 (1998).

${ }^{20}$ T. Hotta, S. Yunoki, M. Mayr, and E. Dagotto, Phys. Rev. B 60, R15009 (1999).

${ }^{21}$ E. Dagotto, T. Hotta, and A. Moreo, Phys. Rep. 344, 1 (2001); A. J. Millis, B. I. Shraiman, and R. Mueller, Phys. Rev. Lett. 77, 175 (1996); P. B. Allen and V. Perebeinos, ibid. 83, 4828 (1999).

${ }^{22}$ J. A. Vergés, V. Martín-Mayor, and L. Brey, Phys. Rev. Lett. 88, 136401 (2002).

${ }^{23}$ J. L. Alonso, L. A. Fernandez, F. Guinea, V. Laliena, and V. Martin-Mayor, Nucl. Phys. B 596, 587 (2001).

${ }^{24}$ G. Alvarez, C. Sen, N. Furukawa, Y. Motome, and E. Dagotto, Comput. Phys. Commun. 168, 32 (2005).

${ }^{25}$ S. Kumar and P. Majumdar, Eur. Phys. J. B 50, 571 (2006). 
${ }^{26}$ S. Kumar and P. Majumdar, Phys. Rev. Lett. 96, 016602 (2006).

${ }^{27}$ J. Salafranca and L. Brey, Phys. Rev. B 73, 214404 (2006).

${ }^{28}$ Y. Motome and N. Furukawa, Phys. Rev. B 68, 144432 (2003).

${ }^{29}$ G. Bouzerar and O. Cépas, Phys. Rev. B 76, 020401(R) (2007).

${ }^{30}$ S. Kumar and P. Majumdar, Eur. Phys. J. B 46, 237 (2005).
${ }^{31}$ B. B. Van Aken, O. D. Jurchescu, A. Meetsma, Y. Tomioka, Y. Tokura, and T. T. M. Palstra, Phys. Rev. Lett. 90, 066403 (2003).

${ }^{32}$ B. M. Andersen, P. J. Hirschfeld, A. P. Kampf, and M. Schmid, Phys. Rev. Lett. 99, 147002 (2007). 